\title{
ENVEJECIMIENTO Y SOLEDAD AGINGANDSOLITUDE
}

\author{
*Álvarez González, R., "Cullell Pares, M., " De Frutos Gallego, E., " ***abretat \\ Casamitjana, MA., *Ramírez Llaras, A.
}

*Diplomada en Enfermería. **Doctora en Pediatría. **Adjunta de Dirección. ABS Raval Nord. CAP Dr. Lluís Sayé. SAP Litoral. Barcelona (ICS).

Palabras clave: Envejecimiento; Soledad; Enfermería en Salud Comunitaria; Urgencias.

Keywords: Aging, Solitude, Nursing in Community Health, and Emergency Unit.

\section{RESUMEN}

Objetivo: Analizar si en las personas mayores en situación de fragilidad que han sido atendidas por el servicio de urgencias domiciliarias, constaba el seguimiento por el personal de enfermería del centro y si constaba el diagnóstico de "Personas mayores en situación de fragilidad".

Material y método: Se realizó un estudio observacional en dos fases: 1' se analizaron todas las personas mayores de 64 años que fueron atendidas en el servicio de urgencias domiciliarias del centro de Atención Primaría de Raval Nord, se analizó si en dicha población constaba el seguimiento de enfermería y el diagnóstico de personas mayores en situación de fragilidad ; $2 a$ fase, se realizó un muestreo simple de la población analizada y se evaluó si se produjeron cambios en los diagnósticos de enfermería y seguimiento de dicha población.

Resultados: De un total de 776 visitas domiciliarias de urgencias médicas, se observó que 568 $(73,19 \%)$ eran mayores de 64 años. Del total, se estudiaron $57(10 \%)$ casos y se observó que el $80 \%$ pertenecían al grupo de edad comprendido entre 75 y 85 años; el $77,26 \%$ eran mujeres; el $94 \%$ con diagnóstico crónico (Diabetes, Hipertensión Epoc...); el 77,2\% eran personas polimedicadas y el $66,7 \%$ del total no estaban incluidas en el programa de Atención Domiciliaria y no constaba ningún diagnóstico de "personas mayores frágiles". En la segunda fase se observa un incremento en la atención por parte de los profesionales de enfermería y un aumento en el diagnóstico de "Personas mayores frágiles".

Conclusiones: La utilización del diagnóstico de enfermería en las personas en situación de fragilidad es una herramienta imprescindible para el seguimiento y control de dicha población. 
Objective: The aim of this study is to analyze whether elderly people in a state of fragility, visited by the Emergency Domiciliary Care service, have received a follow-up program by the nursing staff and whether they were labeled as "Elderly people in state of fragility."

Material and method: A two-phase observational study. Firstly, in phase I, a population comprising people older than 64 and visited by the emergency domiciliary service from the Raval Nord Primary Care Centre was selected. Whether they had received nursing follow-up and whether they had been diagnosed as elderly people in a frail state was analyzed. In phase II, a simple random sample from this population was chosen. Here, it was analyzed whether there had been changes in the nursing diagnosis and follow-up.

Results: Of a total was seen that of 776 medical emergency domiciliary visits, 568 (73.19\%) were people belonging to the over 64 group. Out of the total, 57 cases $(10 \%)$ belonged to the age group between 75 and 85 years old (80\%). Out of this group, $77.26 \%$ were women; $94 \%$ were diagnosed with a chronic condition (diabetes, hypertension, chronic obstructive pulmonary disease, etc); and $77.2 \%$ were polymedicated. In addition, $66.7 \%$ were not included in the Domiciliary Care Program and were not diagnosed as frail elderly people. In phase II, an increase in nursing care, as well as in the "frail elderly people" diagnosis were found.

Conclusions: The use of nursing diagnosis for elderly people in a frail state is an indispensable tool for the monitoring and follow-up of that population.

\section{INTRODUCCIÓN}

En los últimos años la población española presenta un proceso de envejecimiento similar al resto de sociedades llamadas desarrolladas. Según la OMS se calcula que en el año 2025 alrededor de 1000 millones de personas tendrán más de 60 años. Y si el hecho de envejecer no es ninguna enfermedad, y no debemos asociar vejez con dependencia (1), es bien cierto, no obstante, que va acompañado de un aumento de incidencia y prevalencia de enfermedades crónicas cardiovasculares, diabetes, enfermedades de Alzheimer y otras afecciones de salud mental relacionadas con el envejecimiento (2). Esta situación se agudiza en ciudades con alto índice de densidad como la ciudad de Barcelona, que tiene una casco antiguo con una densidad superior a $21.074 \mathrm{hab} / \mathrm{km} 2$, y que es la zona de la ciudad en la que mayor índice de población envejecida hay, el $30 \%$ de su población es mayor de 65 años. Según el estudio "Las Personas mayores en España 2004" (IMSERSO), en el año 2030 en España la población mayor de 80 años será el 7,2\% (2) y el mismo informe nos dice que el $32,4 \%$. de esta población vive en soledad.

La edad avanzada, junto con la soledad, puede ser una causa importante de aislamiento, EI informe realizado por la Agencia de Salud Pública de Barcelona (ASPB) (3), nos dice que en el año 2007 el 40,5\% de las mujeres y el $20 \%$ de los hombres mayores de 85 años viven solos, y que por barrios, en Ciutat Vella, la mitad de las ancianas viven solas y una cuarta parte de hombres también. Esta situación es fácil de observar en el Centro de Salud Raval Nord (CAP) ubicado en el distrito de "Ciutat Vella" de Barcelona. En la historia clínica informatizada (e-cap) del CAP, utilizada en la mayoría de los centros públicos de Atención Primaria de Cataluña, existe el diagnóstico de "Vell Frágil = Persona Frágil" (población frágil). Se incluye en este apartado toda persona mayor de 65 años que cumpla un ítem del test de Barber (4). Asimismo existe el programa de atención domiciliaria (ATDOM) (5) dirigido a toda la población que no pueda desplazarse al CAP por un motivo de salud y requiera atención sanitaria.

A través de estos mecanismos se pretende, entre otras cosas, dar continuidad a la atención sanitaria y seguimiento del estado de salud de toda la población. 
El presente trabajo tiene como objetivo principal analizar si la población de edad avanzada que solicita en el CAP visitas de urgencia domiciliaria tiene seguimiento de los cuidados "se puede vivir sin tratamientos, pero no se puede vivir sin cuidados" (6) por parte del personal de enfermería.

\section{OBJETIVO}

Analizar si la población de edad avanzada que solicita visita de Urgencias en el centro de Atención Primaria tiene seguimiento por parte de los profesionales de Enfermería.

\section{Objetivos específicos}

1) Analizar si a través de la Historia clínica (Hcap) de las personas adultas en situación de fragilidad consta registrado el diagnóstico de "Vell Frágil";

2) Determinar si en esta población consta el diagnóstico de enfermería;

3) Identificar si las personas adultas de riesgo vistas en el servicio de urgencias presentan problemas crónicos de salud en los que ha de intervenir el personal de enfermería en los cuidados,

\section{MATERIAL Y MÉTODO}

Se realiza un estudio observacional, descriptivo, para identificar si las personas mayores en situación de fragilidad que solicitaban servicio de urgencias domiciliarias constaba el registro de su situación de "persona frágil". Se analizó el total de la muestra y se recogieron a través de la Historia Clínica informatizada las distintas variables.

Se realizaron 2 fases: de enero de 2007 a finales de julio de 2007 en la primera fase y una segunda fase en el año 2008 en la que se analizó la misma muestra.

De un total de 776 visitas domiciliarias de urgencias médicas se observó que $568(73,19 \%)$ eran mayores de 64 años y el resto, un total de $208(26,80 \%)$, eran menores.

Se realizó un muestreo aleatorio simple y se obtuvo un total de 57 casos que representan el $10 \%$ de la muestra total.

Las variables objeto de estudio fueron: Edad; Sexo; Diagnóstico de "Persona Frágil"; Registro de diagnóstico de enfermería; Presencia de problemas crónicos de salud, considerando enfermedad cardiovascular, diabetes, Epoc; Polimedicación (mas de cinco fármacos).

Se efectuó una base de datos con las variables definidas y la explotación estadística a través del programa informático Statistical Package for the Social Sciencies ( SPSS). EI estudio de las variables definidas se realizó a través de las Historias Clínicas del programa E-cap.

\section{RESULTADOS.}

En la primera fase se observó que del total de 57 casos estudiados, más del $80 \%$ del total pertenecía a los grupos mayores de 75 años y 85 años, seguido del grupo de más de 65 años que representó el 14\%, según observamos en la tabla 1. 
Tabla 1: Grupos de edad.

\begin{tabular}{|c|c|c|}
\hline Edad & Frecuencia & Porcentaje \\
\hline $\begin{array}{l}65 \text { años i } \\
\text { mes }\end{array}$ & 8 & 14,0 \\
\hline $\begin{array}{l}75 \text { años i } \\
\text { mes }\end{array}$ & 26 & 45,6 \\
\hline $\begin{array}{l}85 \text { años } \\
\text { mes }\end{array}$ & 20 & 35,1 \\
\hline $\begin{array}{l}95 \text { años } i \\
\text { mes }\end{array}$ & 3 & 5,3 \\
\hline Total & 57 & 100,0 \\
\hline
\end{tabular}

En relación a el sexo se observó que el $77,2 \%$ eran mujeres, seguidos de hombres en el $22,8 \%$ (tabla 2)

Tabla 2: Sexo

\begin{tabular}{|l|l|l|}
\hline Sexo & Frecuencia & Porcentaje \\
\hline hombre & 13 & 22,8 \\
mujer & 44 & 77,2 \\
Total & 57 & 100,0 \\
\hline
\end{tabular}

En relación al diagnóstico de patologías crónicas (Hipertensión, Diabetes, Epoc) se observó la mayoría en un porcentaje del $94,7 \%$. (tabla 3 )

Tabla 3: patología crónica.

\begin{tabular}{|ll|l|l|l|l|}
\hline & & Frecuencia & Porcentaje & $\begin{array}{l}\text { Porcentaje } \\
\text { válido }\end{array}$ & $\begin{array}{l}\text { Porcentaje } \\
\text { acumulado }\end{array}$ \\
\hline $\begin{array}{llll}\text { Válido } \\
\text { s }\end{array}$ & si & 54 & 94,7 & 94,7 & 94,7 \\
& no & 3 & 5,3 & 5,3 & 100,0 \\
\hline
\end{tabular}

Se observó que el $77,2 \%$ de los casos estudiados eran personas con polimedicación (5 ó más medicamentos), el resto, el $22,8 \%$ no (tabla 4 )

Tabla 4: poli medicamentos.

\begin{tabular}{|l|l|l|}
\hline & $\begin{array}{l}\text { Frecuenc } \\
\text { ia }\end{array}$ & $\begin{array}{l}\text { Porcenta } \\
\text { je }\end{array}$ \\
\hline si & 44 & 77,2 \\
no & 13 & 22,8 \\
Total & 57 & 100,0 \\
\hline
\end{tabular}


Se analizó si eran personas que estaban incluidas en el programa de Atención Domiciliaría, y se observó que el $66,7 \%$ no están incluidas (tabla 5). Al analizar si estaba incluido el diagnóstico de Vell frágil (Ecap: Z91.8) se observó que en ningún caso se había registrado dicho diagnostico.

Tabla 5: ATDOM

\begin{tabular}{|cc|c|c|}
\hline & & Frecuencia & Porcentaje \\
\hline Válido & si & 19 & 33,3 \\
s & & 38 & 66,7 \\
& no & 57 & 100,0 \\
\hline
\end{tabular}

Se analizó si las personas que acudían a urgencias domiciliarias médicas, habían sido vistas por el personal de enfermería durante los últimos seis meses, observándose que el $68,4 \%$ sí habían sido visitados.

Tabla 6: Visitas realizadas por enfermeria.

\begin{tabular}{|c|c|c|c|}
\hline & & Frecuencia & Porcentaje \\
\hline \multirow{2}{*}{$\begin{array}{l}\text { Válido } \\
\text { s }\end{array}$} & & 39 & 68,4 \\
\hline & $\begin{array}{l}\text { no } \\
\text { Total }\end{array}$ & $\begin{array}{l}18 \\
57\end{array}$ & $\begin{array}{l}31,6 \\
100,0\end{array}$ \\
\hline
\end{tabular}

En relación al diagnóstico de enfermería, se observó que en el $68,4 \%$ de los casos no había diagnóstico de enfermería. (Tabla 7)

Tabla 7: Diagnósticos de enfermería

\begin{tabular}{|ll|l|l|}
\hline & & Frecuencia & Porcentaje \\
\hline Válido & sí & 18 & 31,6 \\
s & & 39 & 68,4 \\
& no & 39 & 100,0 \\
\hline \multicolumn{2}{|c|}{ Total } & 57 & \\
\hline
\end{tabular}

Al analizar si en los últimos seis meses fueron visitados por el profesional de enfermería, se observó que el $68,4 \%$ sí habían sido visitados, y el $31,6 \%$ no.

De este porcentaje del $31,6 \%$ de diagnóstico de enfermería registrado se observa que la mayoría $(21,1 \%)$ de los diagnósticos se refieren a las personas incluidas en el programa. ATDOM. (Tabla 8) 
Tabla 8:

Tabla de contingencia diag_infer * atdom

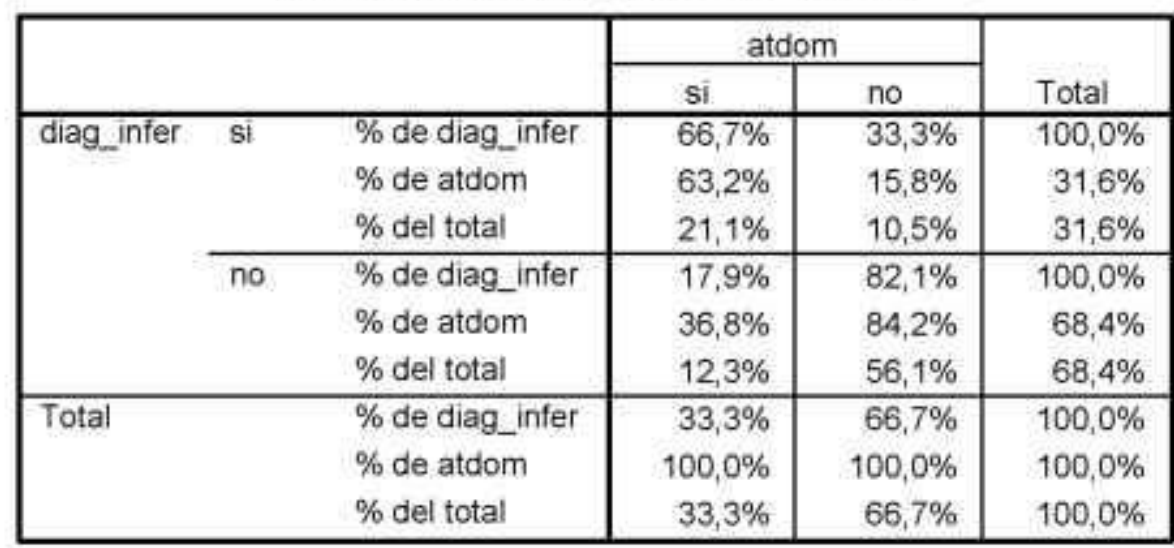

\section{SEGUNDA FASE}

Al analizar después de transcurrido un año el comportamiento de dicha muestra, se observó que el total de 57 casos se reduce a 49 casos, por motivos de exitus, ingresos en residencia y traslados domiciliarios.

Del total de 49 casos se observó que el diagnóstico de Enfermería representaba el 40,4\%, un ligero aumento de 23 casos en el 2008 en relación a la tabla 7 que eran 18 casos (El $33,3 \%$ ) observados en el análisis del año 2007. (Tabla 9)

Tabla 9 : Diagnósticos de enfermeria

\begin{tabular}{|ll|l|l|}
\hline & & Frecuencia & Porcentaje \\
\hline Válidos & si & 23 & 40,4 \\
& no & 26 & 45,6 \\
& Total & 49 & 86,0 \\
Perdido & Sistem & 8 & 14,0 \\
s & a & 57 & 100,0 \\
Total & & 57 & \\
\hline
\end{tabular}

También se observó que el diagnostico "Vell frágil" representaban el 19,3\% en el año 2008, en cambio en el estudio anterior no constaba en ningún caso diagnosticado el "Vell frágil" (tabla 10) 
Tabla 10: Vell_fragil

\begin{tabular}{|ll|l|l|l|l|}
\hline & & Frecuencia & Porcentaje & $\begin{array}{l}\text { Porcentaje } \\
\text { válido }\end{array}$ & $\begin{array}{l}\text { Porcentaje } \\
\text { acumulado }\end{array}$ \\
\hline Válidos & sí & 11 & 19,3 & 22,4 & 22,4 \\
& no & 38 & 66,7 & 77,6 & 100,0 \\
Perdido & Total & 49 & 86,0 & 100,0 & \\
s & a & 8 & 14,0 & & \\
Total & & 57 & 100,0 & & \\
\hline
\end{tabular}

\section{DISCUSIÓN Y CONCLUSIONES.}

Se observó que la mayoría de las personas que solicitaron urgencias domiciliarias tenían más de 65 años, siendo el grupo de edad entre 75 a 85 años el de más solicitantes (7). En su mayoría estaban diagnosticados con alguna patología crónica, polimedicados y no incluidos en el programa de atención domiciliaria.

En el primer periodo del estudio sorprendió que no hubiera en ningún caso de la muestra registrado con el diagnostico de "Vell frágil" y que los diagnósticos de enfermería sólo se habían registrado en las personas que estaban incluidas en el programa de atención domiciliaria (ATDOM) (5). En cambio en el segundo análisis correspondiente al año 2008, se observó una mayor captación de las personas mayores. Había un aumento en el diagnóstico de "personas frágiles" y aumentaban los diagnósticos de enfermería en la población autónoma que acudía a las consultas de forma independiente, probablemente por la sensibilización de los sanitarios al comunicar la realización de este estudio.

La población en situación de fragilidad es la que de forma preferente debe de estar controlada por el personal de enfermería, ya sea en el domicilio o en la consulta, ya que tienen una serie de problemas derivadas de su patología que son competencia del personal de enfermería (8). Las personas incluidas en el programa ATDOM es más frecuente que tengan un seguimiento de sus problemas de salud ya que a través de los programas informáticos se pueden realizar listados y comprobaciones del seguimiento de estos pacientes. Existe un protocolo que indica la periodicidad que se debe seguir para realizar la visita domiciliaria en función de la patología o situación. En cambio en las personas mayores en situación de fragilidad, si no se hace constar el diagnóstico de "persona frágil", si tras la vista de urgencia nos limitamos simplemente a comunicarle el día de la próxima visita, puede existir el riesgo de que no se solicite o no acudan a la misma, ya sea por olvido, pérdida de memoria, soledad, aislamiento, etc. Esta situación nos debe llevar a pensar en un circuito que nos ayude y facilite el seguimiento de esta población.

\section{CONCLUSIONES}

Establecer y codificar el diagnóstico de "persona frágil" podría ser una buena herramienta, para que a través de los programas informáticos se realicen listados y comprobaciones que ayuden al seguimiento de las personas en situación de fragilidad, pues en muchas ocasiones el contacto con los servicios de salud sólo se realiza a través de la demanda a los servicios de urgencias. 


\section{BIBLIOGRAFÍA}

1. Rodríguez Borrego Ma A. La Ancianidad en las publicaciones periódicas enfermeras: Rol y Nursing edición española, de 1997 a 2000. Rev. Enfermería Global, $n^{\circ}$ 10, mayo 2007. Acceso en: http://revistas.um. es/eglobal

2. Imserso .Envejecer en el SXXI. Boletín sobre el envejecimiento. Perfiles y tendencias. Febrero 2006 .

Acceso en : www.seg-social.es/imserso/masinfo/boletinopm21.pdf

3. Resultados: de l'informe "La salud a Barcelona 2007" :Una ciutat amb bona salud.Agencia de Salud Pública de Barcelona (ASPB) . 19 Noviembre 2007. Accesible en: http://www.aspb.es/quefem/docs/ndp informe\%20de\%20salut 08.pdf

4. Cuestionario de Barber (detección de anciano de riesgo) 1. ¿Vive solo?. En Barber $\mathrm{JH}$, Wallis JB, McKeating $\mathrm{E}$. A postal screening questionnaire in preventive geriatric care. J R Coll Gen Pract 1980,. $30 \quad$ (210): 49-51. Accesible en: www.sld.cu/galerias/pdf/sitios/rehabilitacion-doc/anciano_de_riesgo.pdf

5. Ministerio de Sanidad y Consumo.Instituto Nacional de la Salud.Dirección de Atención Primaria y Especializada.Programa de Atención domiciliaria con equipos de soporte (ESAD).Madrid, 1999.

6. Ma Lourdes Cobacho Inglés. Dependencia y Vejez. Una Aproximación Al Debate Social [Revisión y reseña]. Enfermería Global. Mayo $2007 . \wedge 11$

7. Segura Noguera JM, Bastida Bastús N, Martí Guadaño N, Riba Romera M, Marsá Cantero M, Lancho Lancho S. Características y análisis de la supervivencia de las personas atendidas en el programa de atención domiciliaria del área básica de salud raval nord, Barcelona ,1993-2002 . Rev Esp Salud Publica .Oct 2003 ; 77 (5)

Acceso en: $\quad$ http://www.scielosp.org/scielo.php?pid=S1135$\underline{57272003000500007 \& \text { script }=\text { sci } \text { arttext }}$

8. Maestro Castelblanque E. Albert Cuñat V.¿Quiénes son ancianos frágiles-ancianos de riesgo?Estudio en personas mayores de 65 años del Área Sanitaria de Guadalajara . Medicina General. 2002 ; 47: 667-680.

Acceso en: http://www.medicinageneral.org/revista 47/pdf/667-680.pdf

\section{ISSN 1695-6141}

